\title{
Extracellular vesicles, from pathogenesis to biomarkers: the case for cerebral malaria
}

\author{
Iris S. Cheng ${ }^{1, \#,}$, Benjamin C. Sealy ${ }^{1, \#,}$, Natalia Tiberti ${ }^{2}$, Valery Combes ${ }^{1}$ \\ 'Faculty of Science, School of Life Sciences, University of Technology Sydney, Sydney 2007, Australia. \\ ${ }^{2}$ Department of Infectious - Tropical Diseases and Microbiology, IRCCS Sacro - Cuore Don Calabria Hospital, Verona Area 37024, \\ Italy. \\ "Co-equal first authors.
}

Correspondence to: Associate Prof. Valery Combes, Faculty of Science, School of Life Sciences, University of Technology Sydney, Australia. E-mail: valery.combes@uts.edu.au

How to cite this article: Cheng IS, Sealy BC, Tiberti N, Combes V. Extracellular vesicles, from pathogenesis to biomarkers: the case for cerebral malaria. Vesse/ Plus 22020;4:17. http://dx.doi.org/10.20517/2574-1209.2020.08

Received: 5 Feb 2020 First Decision: 5 Mar 2020 Revised: 1 Apr 2020 Accepted: 7 Apr 2020 Published: 16 Jun 2020

Science Editor: Narasimham L. Parinandi Copy Editor: Jing-Wen Zhang Production Editor: Jing Yu

\begin{abstract}
Malaria infections due to the Plasmodium parasite remains a major global health problem. Plasmodium falciparum is responsible for majority of the severe cases, resulting in more than 400,000 deaths per annum. Extracellular vesicles (EVs) released by vascular cells, including parasitised erythrocytes, have been detected with increased levels in patients with malaria. EVs are thought to be involved in the pathogenesis of severe malaria, particularly cerebral malaria, and represent a unique molecular signature for different forms of the infection. In this review, we will cover the known effects of EVs on the vasculature and discuss their potential use as a biomarker of disease severity.
\end{abstract}

Keywords: Cerebral malaria, extracellular vesicles, biomarker, pathogenesis, microvesicles, exosomes

\section{INTRODUCTION TO MALARIA}

Malaria can be a life-threatening disease and remains a global health problem with an estimated incidence of 228 million cases and 405,000 deaths in $2018^{[1]}$. While its incidence has decreased significantly in the last 15 years, progress has stalled and case numbers are starting to increase again in some countries with drug resistance a major threat ${ }^{[1]}$. Plasmodium falciparum (P. falciparum) is one of six Plasmodium species, all of which can cause disease in humans, and is associated with the development of severe disease. 
Clinically, malaria can be either uncomplicated or severe. Uncomplicated malaria presents as a nonspecific, flu-like syndrome and diagnosis is based only on clinical features, which is often unreliable. Approximately $1 \%$ of diagnosed cases will progress to severe malaria for reasons that are not fully understood $^{[2]}$ and amongst these, up to $30 \%$ will be at risk of developing life-threatening or debilitating complications $^{[3]}$. Severe malaria is defined by precise diagnostic criteria related to specific signs and symptoms, with cerebral malaria (CM) and severe malarial anaemia (SMA) being two of the most serious life-threatening complications associated with P. falciparum infection. Both target children under the age of five and although not yet fully understood, the pathogenesis of CM and SMA is likely to be different. The clinical hallmark of CM is a diffuse, symmetrical encephalopathy with coma and a general absence of focal neurological signs. CM is characterised by the sequestration (binding) of infected red blood cells (iRBCs) in the vasculature of most organs, including the brain, coupled with an uncontrolled inflammatory response $^{[4]}$. This sequestration of iRBCs during CM is associated with endothelial dysfunction leading to coma, respiratory distress syndrome and placental malaria when it occurs in the brain, lungs or during pregnancy, respectively. SMA is defined by a haemoglobin $(\mathrm{Hb})$ concentration $<5 \mathrm{~g} / \mathrm{dL}$ and a packed cell volume $(\mathrm{PCV})<15 \%$ in children, and by $\mathrm{Hb}<7 \mathrm{~g} / \mathrm{dL}$ and $\mathrm{PCV}<20 \%$ in adults ${ }^{[5]}$. SMA is also associated with increased clearance of both iRBCs and non-infected red blood cells (nRBCs), as well as altered haematopoiesis ${ }^{[6-8]}$. In both CM and SMA cases however, iRBCs remain within the vasculature, adhere to and activate endothelial cells that are then likely to release pathogenic factors into the surrounding tissues such as the brain parenchyma. This review will focus mainly on CM and its association with extracellular vesicles.

\section{PATHOGENESIS OF CM: FROM HOST CELLS TO EXTRACELLULAR VESICLES}

As mentioned above, CM is characterised by sequestration of iRBCs within the cerebral vasculature although the neurological lesion extends beyond blood vessel alteration to damage to the brain parenchyma, with clear involvement of the blood-brain barrier (BBB). There is a fine and complex interplay between the cells on each side of the BBB, with vascular cells, (i.e., endothelial cells, platelets, T cell lymphocytes, macrophages and to lesser extent neutrophils), microglial cells, neurones, and astrocytes, all having either target or effector roles (and sometimes both) at some point in disease development ${ }^{[9]}$. In addition, extracellular vesicles (EVs) are potentially released by all these cells adding another level of complexity to this intercellular crosstalk. A combination of ex vivo studies using patient samples (biological fluids or post-mortem tissues), in vitro assays mimicking the intravascular lesion, and in vivo experiments using mostly murine models allows for a better understanding of the cellular interactions and pathogenesis of the disease.

How much of CM is attributable to the sequestration of iRBCs is still unknown. Post-mortem studies have shown various levels of $\mathrm{iRBC}$ accumulation within the microvasculature of the brain in patients with diagnosed CM, but this was similarly observed in patients who died of non-CM causes ${ }^{[10,11]}$. Of note, this observation is correlated with the severity of the disease in both children and adults ${ }^{[10,12]}$. Post-mortem histopathology in Malawian children with clinically defined CM (coma and P. falciparum parasitaemia) identified different disease patterns: (1) iRBCs sequestration only; (2) iRBCs sequestration with associated peri-vascular changes such as haemorrhages or micro-thrombi; and (3) little to no sequestration ${ }^{[11]}$. In the latter, the real cause of death was only identified after autopsy, adding to the complexity of CM and the difficulty in establishing a precise diagnosis. In this study ${ }^{[11]}$, only fundus examination allowed discrimination between malarial and non-malaria coma. In Vietnamese adults, iRBC sequestration was more frequent in patients with CM than in those without, and was correlated with coma and time of death $^{[12]}$. Consequently, vascular congestion was proposed as a cause for coma since sequestration leads to decreased cerebral blood flow, impaired brain function and cerebral hypoxia. 
Sequestration occurs via the binding of parasite-related ligands, expressed on the surface of iRBCs, to receptors on the surface of vascular endothelial cells. P. falciparum erythrocyte membrane protein 1 (PfEMP1) is one such molecule expressed on the surface of iRBCs that then binds to a series of endothelial receptors such as CD36, intercellular cell adhesion molecule-1 (ICAM-1), vascular cell adhesion molecule-1, P-selectin, E-selectin, endothelial protein $\mathrm{C}$ receptor or thrombospondin. The expression of these receptors is further modulated by pro-inflammatory cytokines such as tumour necrosis factor (TNF) or interferon-gamma (IFN- $\gamma$ ), thereby supporting inflammation as a critical player in the regulation of sequestration $^{[13,14]}$.

Together with iRBCs and nRBCs, platelets also play an important role in $\mathrm{CM}^{[15]}$. Thrombocytopenia is a hallmark of CM but whether platelet counts can be predictive of lethality in CM is still controversial ${ }^{[16,17]}$. Platelets were found in high numbers in vascular lesions of the brain of Malawian children who succumbed to $\mathrm{CM}^{[18]}$ and are thought to have contributed to the severity of the disease through clumping ${ }^{[19]}$, activation of endothelial cells ${ }^{[20]}$ or increased sequestration via the transfer of $\mathrm{CD} 36$ to brain endothelial cells that are otherwise devoid of $\mathrm{it}^{[21]}$. On the other hand, platelets also are thought to have a protective role during CM by killing intra-erythrocytic parasites ${ }^{[22,23]}$. Therefore, platelets could have different roles at different stages of the disease, i.e., a protective role during the early phase of disease and a pathogenic role when severe ${ }^{[24]}$.

The $\mathrm{BBB}$ is at the centre of the neurovascular lesion occurring in CM although iRBCs do not actively cross this barrier as seen in other pathogens with brain tropism ${ }^{[25,9]}$. Post-mortem histopathological brain studies have demonstrated impairment of the $\mathrm{BBB}$ which suggests that the localised sequestration of iRBCs increases the pressure within microvessels to act on cellular tight junctions, thereby altering the permeability of the BBB which results in micro-haemorrhages when these junctions rupture. Neurological sequelae observed in children who have recovered from CM are also suggestive of neuronal damage ${ }^{[26]}$. Neuronal dysfunction is likely an indirect consequence of the sequestration of iRBCs, activation of the endothelium, alteration of junctional permeability and passage of cytokines, chemokines and other inflammatory mediators into the perivascular space ${ }^{[27,28]}$. However, in most cases, it is likely that these alterations are localised, as symptoms are quickly reversed once parasites have been eliminated. More recently, Magnetic Resonance Imaging has been successfully used as a non-invasive way to predict fatal outcomes in paediatric CM, notably in Malawi ${ }^{[29,30]}$.

As mentioned earlier, crosstalk between vascular cells, including immune and brain parenchyma cells, via direct contact, soluble mediators and molecules leaking through the $\mathrm{BBB}$, can all contribute to the neurological syndrome. In addition, subjecting all these cells to various stimuli can lead to the release of EVs that in turn, target other cells distant from their site of production. Long considered as inert cellular debris, EVs are now accepted as biological effectors in many infectious and inflammatory diseases including malaria ${ }^{[31,32]}$.

EVs represent an ensemble of membrane-bound structures grouped into three main categories: exosomes, microvesicles, and apoptotic bodies. This nomenclature can vary and the term EVs usually encompasses subpopulations of vesicles ranging in size from $30 \mathrm{~nm}$ to $4 \mu \mathrm{m}$, i.e., exosomes produced by membrane invagination of multivesicular bodies, microvesicles (MVs) released after budding of the plasma membrane, or apoptotic bodies that result from blebbing of the plasma membrane of apoptotic cells ${ }^{[33]}$. It is now clear that the role of EVs goes far beyond simple structural function to active mediators of important biological processes for parasitic infections such as immunomodulation, parasite virulence, target cell invasion and parasite-parasite communication ${ }^{[34,35]}$.

In malaria infection, two categories of EVs (i.e., exosomes and microvesicles) have been studied the most. While known in other illnesses for several decades, MVs in malaria patients were first described by our 
group in 2004 in Malawian children with $\mathrm{CM}^{[36]}$, where an elevation in the number of MVs of endothelial origin was described. MVs released by RBCs were later found to be increased in both $P$. falciparum and P. vivax malaria ${ }^{[37,38]}$. EVs have been shown to be involved throughout the entire life-cycle of malaria infection and at different stages, the parasite can affect various immune and vascular cell types in different ways, ultimately altering the endothelium and BBB function ${ }^{[32]}$.

A pan-vascular, cell-derived MV release was also observed in children with CM in Cameroon. Of these MVs, an increase in platelet MVs was most significantly correlated with disease severity ${ }^{[39]}$. Exosomes were first explored in 2011 in a murine model of malaria ${ }^{[40]}$ and will be discussed in a later section. While mostly descriptive, these clinical studies were essential for suggesting a role for these EVs as either markers of CM severity, or as players in the pathogenesis of CM infection, and paved the way for subsequent work on the composition and functional potential of EVs during malaria infection.

\section{IN VITRO MODELS OF MALARIA - INTERACTIONS BETWEEN HOST CELLS AND EXTRACELLULAR VESICLES}

Most in vitro models of CM simulate the interactions between microvascular endothelial cells and circulating vascular cells (e.g., iRBCs, nRBCs, platelets, and leucocytes) in either static or shear stress environments $^{[41,21]}$. The brain endothelial cells used can be of human, simian or murine origin (primary or immortalised), and co-cultured with one or more other cell types in two-dimensional systems ${ }^{[42-45]}$. The recent introduction of more complex three-dimensional models will help to examine and understand the pathogenesis of this disease better ${ }^{[46-48]}$.

Very much like their cells of origin, EVs interact with their target cells and modulate their responses. In vitro, platelet MVs behave in a similar fashion as platelets by increasing the adherence of iRBCs to human brain endothelial cells (HBECs) by providing iRBCs with surface receptors such as CD31 and CD 36 ${ }^{[49]}$ such that platelet MVs act as a bridge between HBECs and iRBCs. The internalisation of platelet MVs by vascular endothelial cells is also associated with an alteration of their phenotype such that ultimately, their inflammatory effects and subsequent activation can be potentiated ${ }^{[50]}$.

RBCs release increased levels of EVs when infected with a Plasmodium parasite and late-stage infections are associated with even greater release of $\mathrm{EVs}^{[38]}$. This is mainly due to membrane changes occurring within iRBCs during parasite maturation. The composition of EVs derived from iRBCs is also dependent on the parasite's stage of development. Indeed, specific parasite proteins, considered as virulence factors, were present in EVs only at specific developmental stages and PfEMP1 was only detected in EVs from iRBCs with parasites at early stages. Potentially, such developments would allow EVs to bind and prime endothelial cells for later adherence and sequestration of late-stage $i \operatorname{RBCs}{ }^{[51]}$.

EVs from iRBCs have also been shown to contain a functional microRNA-argonaute 2 complex that can modulate gene expression and alter barrier function ${ }^{[52,53]}$ when transferred to endothelial cells after vesicle uptake. Such EVs do not only affect endothelial cells but are also able to induce pro-inflammatory responses, particularly the activation of macrophages, monocytes as well as other immune cells through the upregulation of cytokines ${ }^{[54,55]}$. Interestingly, when these EVs were compared to their mother cells, they were able to activate inflammation and immune activation to a greater degree ${ }^{[54,55]}$. EVs from iRBCs also contain small RNAs and genomic DNA. After internalisation by monocytes, they can induce the innate immune cytosolic adaptor-dependent DNA sensing pathway (STING), leading to downstream alterations of DNA sensing pathways in target cells ${ }^{[56]}$. Activation of these pathways has been shown to correlate with parasite survival $^{[57]}$. Thus, this could possibly be used as a decoy method for immune escape by the parasites ${ }^{[57]}$. Similarly, the release of PfEMP1-containing EVs as previously mentioned, has also been suggested as a 
decoy strategy as it is capable of inducing both the production of inflammatory cytokines (IL-12, CCL2, and CCL4) by monocytes after internalisation and transcriptomic changes ${ }^{[51]}$. Furthermore, as the majority of the body's natural immune response to P. falciparum targets PfEMP1, the secretion of PfEMP1containing EVs could possibly work as a smokescreen by attracting neutralising antibodies that protect the parasite from the immune system ${ }^{[13,51]}$. EVs can also mediate immunosuppression in mice infected with malaria with EVs from $P$. berghei-iRBCs able to inhibit $\mathrm{CD}^{+}{ }^{+} \mathrm{T}$ cell proliferation in response to antigen presentation. This process seems to be mediated by two potential virulence factors, histamine-releasing factor and elongation factor $1 \alpha(\mathrm{EF}-1 \alpha)$. Importantly, this work also showed that mice immunisation with EVs from $P$. berghei-iRBCs or recombinant $P$. berghei-EF-1 $\alpha$ resulted in resistance to infection, further suggesting the role of EVs in immune-modulation and potential for vaccine development ${ }^{[58]}$.

Exosome-like vesicles derived from iRBCs have been reported to facilitate communication between iRBCs and therefore, promoting gametocytogenesis between parasites in vitro via the transfer of a $P$. falciparum protein ${ }^{[59]}$. This communication is also used to improve parasite survival within the host as well as transmission to mosquitoes.

Although not specifically studied in an in vitro model of CM, endothelial MVs interacting with $\mathrm{T}$ lymphocytes have been found to assist cell proliferation by inducing cell activation and antigen presentation by immune cells ${ }^{[60]}$. In addition, when MVs from lipopolysaccharide-stimulated monocytes are internalised by HBECs, they release high levels of MVs (usually a sign of cell activation) and at the same time, display an increase in trans-endothelial resistance (i.e., tightening of endothelial junctions) which could have a protective effect on the BBB if occurring in vivo. This suggests that MVs from monocytes, as was shown for MVs from neutrophils, could trigger contrasting protective and pathogenic responses ${ }^{[61-63]}$.

In vitro models of malaria are limited in their ability to mirror the pathogenesis of CM and more complex systems are needed to understand the fine interplay between host cells and EVs during malaria infection.

\section{IN VIVO MODELS OF MALARIA: WHAT DO EXTRACELLULAR VESICLES BRING TO PATHOGENESIS?}

Although there is still debate regarding the usefulness of murine models for studying the pathogenesis of CM, human studies are limited and often, post-mortem analyses are the only way to explore some parameters. However, the number of studies that find parallels between human and experimental CM (ECM) continues to grow. Most recently a study ${ }^{[64]}$ observed that in post-mortem cases of paediatric $\mathrm{CM}, \mathrm{CD}{ }^{+} \mathrm{T}$ cells were found within both the vascular lumen as well as the juxtavascular space as was previously shown in murine $\mathrm{CM}$ studies ${ }^{[65,66]}$. Therefore, animal models can still provide relevant basic scientific knowledge and allow testing of important hypotheses related to the pathogenesis of the disease ${ }^{[67-71]}$. For instance, whole-animal imaging using transgenic fluorescent parasites has demonstrated that sequestration, and not only accumulation, of iRBCs, does occur in all organs similar to humans ${ }^{[72-74]}$. In addition, recent quantitative mapping of mice brains during ECM showed similar numbers compared to human CM despite the distinct aetiology ${ }^{[75]}$. However, as for any model, it is not perfect and should be used with caution and one should be aware of its limitations before drawing direct conclusions with human disease.

Two different Plasmodium species are commonly used in CM models, P. yoelii ${ }^{[40]}$ and P. berghei $i^{[76]}$, notably $P$. berghei ANKA ( $\mathrm{PbA})$. During the acute phase of infection, mouse strains that are susceptible to $\mathrm{CM}$ (e.g., $\mathrm{CBA} / \mathrm{J}, \mathrm{C} 57 \mathrm{BL} / 6, \mathrm{DBA} 1$ ) display increased levels of plasma MVs similar to that observed in humans ${ }^{[76-78]}$. We examined the ATP-binding cassette transporter A1, which modulates the distribution of phosphatidylserine to the outer leaflet of the cell plasma membrane at the time of MV production ${ }^{[76]}$. We found that mice lacking this ATP-binding cassette had resistance to the malaria-associated neurological 
syndrome in $\mathrm{C} 57 \mathrm{BL} / 6$ mice ${ }^{[76]}$. These animals displayed basal levels of plasma MVs but numbers failed to increase following infection (as observed in wild type counterparts), had lower levels of plasma TNF, reduced expression of endothelial cell adhesion molecules and had increased survival of leukocytes and platelets. Another study examining the blocking of phosphatidylserine using low-molecular-weight thiol pantethine found a similar reduction in MV production, which correlated with reduction in inflammation and resistance to the disease ${ }^{[78]}$. When passively transferred into the circulation of mice, plasma MVs from infected animals localised to the inflamed vessels of infected animals, notably in the brain, which suggests that they could potentiate the neurovascular lesions by interacting with other vascular cells. In addition, healthy mice injected with TNF-generated endothelial MVs developed CM-like pathology with cerebral and pulmonary oedema and haemorrhage, the two main histopathological features of human and murine $\mathrm{CM}^{[77,79,80]}$.

Interaction with and internalisation of MVs derived from iRBCs by astrocytes and microglial cells leads to increased production of IFN- $\gamma$-inducible protein 10 (IP-10), which coincided with increased levels of inflammatory cytokines within both plasma and brain tissue of PbA-infected mice ${ }^{[81]}$. Plasma MVs from $\mathrm{PbA}$-infected mice were also able to activate immune cells, in particular macrophages, leading to the upregulation of $\mathrm{CD} 40$ as well as TNF production ${ }^{[54]}$. Knock-out mice that lack pro-inflammatory cytokines $\left(\mathrm{TNF}^{-\nu^{-}}, \mathrm{IFN}-\gamma^{-\gamma^{-}}, \mathrm{IL}_{-1} 2^{-\gamma_{-}}\right.$and RAG-1 ${ }^{-{ }^{-}}$) displayed levels of plasma MVs similar to those of their wild-type counterparts ${ }^{[54]}$, suggesting that their production is not solely dependent on the presence of inflammation.

When exosomes, purified from the blood of $P$. yoelii (nonlethal strain,17XNL)-infected mice, were injected into mice infected with P. yoelii (lethal strain, 17XL), these mice were protected against the lethal syndrome, showing that exosomes could also modulate the immune response ${ }^{[82,40]}$. We propose a model in Figure 1 that summaries the role EVs play in the pathogenesis of CM.

Figure 1 was designed by modifying free images provided by Smart Servier Medical Art (https://smart. servier.com/) and available under the creative commons license.

\section{EXTRACELLULAR VESICLE CARGO: EFFECTOR, BIOMARKER OR BOTH?}

Biomarkers have long been used as diagnostic and prognostic tools to determine the presence of disease as well as the regression, progression or outcome after treatment ${ }^{[85]}$. The field of malaria, especially severe malaria, currently lacks reliable markers for the prediction of morbidities such as neurocognitive impairment and/or mortality that can be widely applicable regardless of country or endemicity. Such biomarkers would allow for the prediction of severe complications and allow early implementation of adjunctive therapies. Current adjunctive therapies used to aid anti-malarial drugs have so far been ineffective ${ }^{[86]}$, possibly due to late implementation due to the lack of predictive biomarkers. Therefore, early identification of patients at risk of severe malaria complications (lethal or not) would allow for prompt treatment and potentially decrease the risk of long-term disabilities.

Currently, only a handful of candidate markers have been identified for severe malaria, though they are not fully reliable. The proposed molecular markers include erythropoietin, angiopoietin 2, von Willebrand factor, P. falciparum histidine-rich protein 2 and ICAM-1 which, although indicative of severe disease when elevated, still has limited clinical utility. This is mainly due to the highly variable sensitivity and specificity for the detection of severe $\mathrm{CM}^{[41,87-90]}$. It is now clear that EVs and their cargo have potential as biomarkers. Their elevated numbers, notably endothelial-, platelet- and RBC-derived MVs, in the circulation of human patients with CM has already been proven in multiple studies ${ }^{[39,84,91]}$. The role of EVs as a biomarker in severe malaria is still in its infancy and in-depth multi-centre studies are still needed to ascertain their predictive value to improve rapid detection in bodily fluids. In addition, although blood, urine, and saliva have all been used for diagnostics ${ }^{[92,93]}$, urine and saliva have not been investigated in malaria but we 


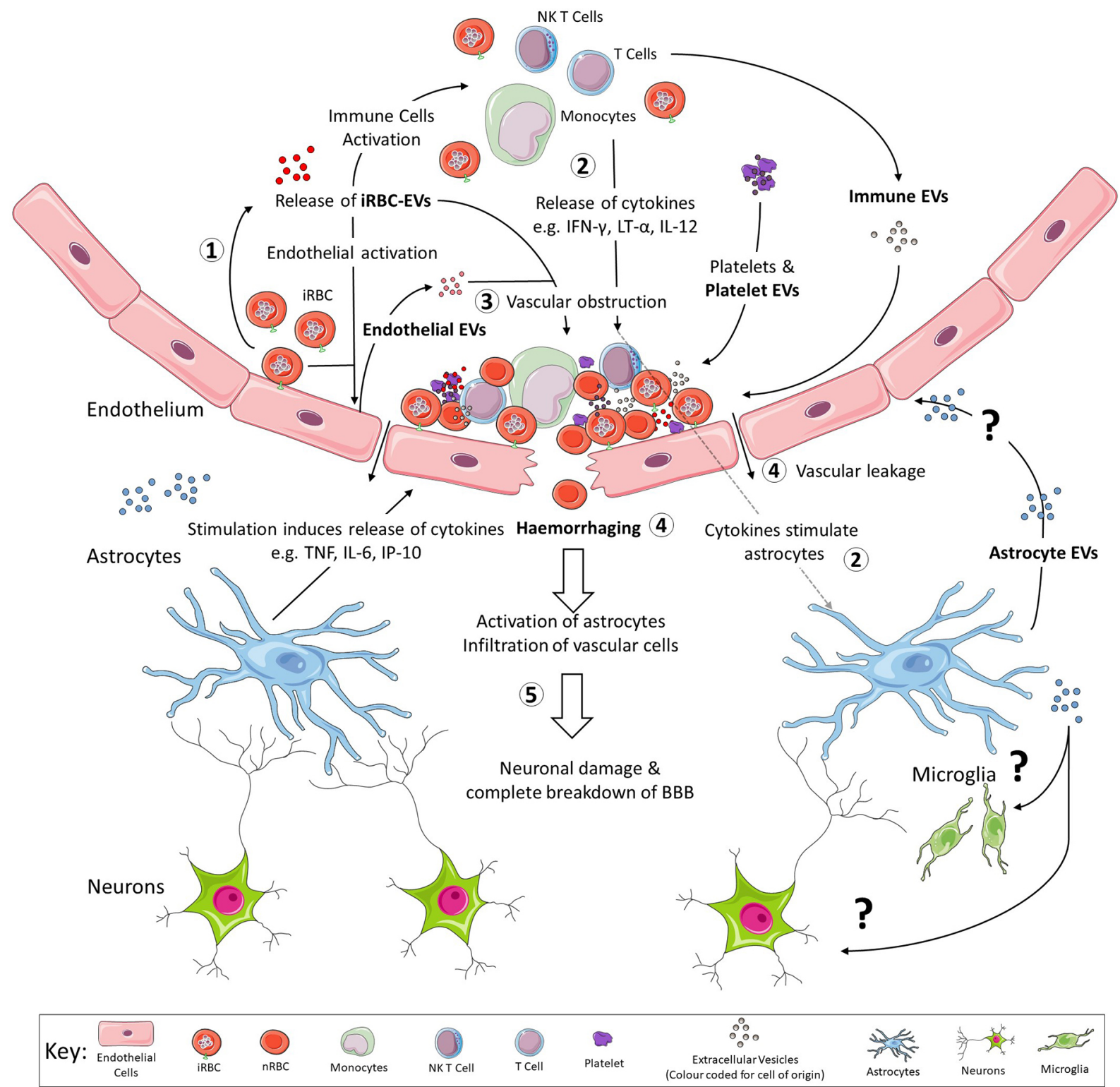

Figure 1. Outline of the role that EVs play in the pathogenesis of cerebral malaria. (1) As iRBCs adhere to vascular ECs, they are also releasing EVs into the blood. These EVs from iRBCs stimulate all vascular cells including immune cells, ECs and platelets to release EVs of their own ${ }^{[83]}$ as reviewed by Babatunde et $a{ }^{[84]}{ }^{84}$. Both ECs and platelet EVs have been shown to assist with the formation of neurovascular lesions by providing mechanisms of binding for iRBCs and other vascular cells as reviewed by El-Assaad et al. ${ }^{[77]}, 2014$ and Faille et $a / .{ }^{[49]}$. (2) EVs from iRBCs have been suggested to act not only as a decoy, by providing alternative targets expressing PfEMP-1 for immune cells to attack ${ }^{[51]}$, but also promote secretion of increased levels of pro-inflammatory cytokines, notably IFN- $\gamma$, LT- $\alpha$ and IL-12 once internalised by immune cells ${ }^{[54,55]}$. These cytokines have also been shown to stimulate astrocytes, which then respond by secreting additional cytokines and chemokines of their own ${ }^{[13]}$. The effect of astrocyte EVs have not been studied in humans but these EVs could have effects on both endothelial cells and cells of the brain parenchyma. (3) The release of these pro-inflammatory cytokines and chemokines further activates the already stimulated ECs, leading to greater adherence of vascular cells and ultimately, formation of the neurovascular lesion. (4) Once the neurovascular lesion has formed, increased intravascular pressure on the endothelium leads to vascular leakage and ultimately results in haemorrhage. (5) The infiltration of vascular cells and cytokines or chemokines causes neuronal damage and subsequently, a localised breakdown of the blood brain barrier ${ }^{[32]}$. EVs: extracellular vesicles; ECs: endothelial cells; iRBCs: infected red blood cells; nRBC: non-infected red blood cell; BBB: blood-brain barrier; TNF: tumour necrosis factor; IFN- $\gamma$ : interferon-gamma; NK: natural killer; LT- $\alpha$ : Iymphotoxin alpha; IL: Interleukin; IP-10: IFN- $\gamma$-inducible protein 10

cannot exclude the possibility of them becoming a source of biomarkers to assess disease severity in the future. As mentioned above, all current markers have their limitations and one could hypothesise that the 
combination of these existing biomarkers with newly discovered EV-associated markers could significantly improve both the specificity and sensitivity of testing.

As a consequence of their biogenesis, EVs harbour a peculiar set of proteins, nucleic acids, and lipids that can be transferred from a parent to recipient cells, rendering these sub-micron structures unique sources and vehicles of biomarkers. Various analytical approaches including proteomics, transcriptomics, and metabolomics, although mostly focused on cancer-related conditions, are currently employed to study the content of EVs derived from different cell types and bodily fluids ${ }^{[94]}$. Nonetheless, the cargo of EVs is now becoming an important research topic in severe malaria allowing us to both understand disease pathogenesis and identify novel biomarkers, with proteins and microRNA (miRNA) being the most studied components of this cargo.

EV-associated proteins can typically be studied using either untargeted proteomics, to characterise the whole protein content, or through a hypothesis-driven targeted approach, to investigate individual proteins or a selected set of proteins based on previous evidence. Compared to other parasitic diseases, highthroughput untargeted proteomics - the leading technique for the discovery of new protein markers has not been widely applied to investigate malaria-associated EVs yet, but has been explored in the last couple of years. The first report dates back to 2011 when exosomes from P. yoelii-infected BALB/c mice were analysed and revealed to contain both classical exosomal markers as well as parasite proteins ${ }^{[40]}$. Interestingly, 30 parasite proteins belonging to two major classes, proteins associated with RBCs membrane and proteins involved in parasite invasion into RBCs, were identified within iRBCs exosomes. Then, the presence of Plasmodium proteins within EVs from human and mice malaria infection was confirmed by a number of proteomics-based studies ${ }^{[40,51,58,82,95]}$. Although the majority of these studies did not have as their main objective the identification of biomarkers, they all contributed to prove the presence of parasitederived proteins with antigenic and immunomodulatory properties, or as potential virulence factors within EVs that, in the future, might be found useful for the development of novel diagnostic and prognostic tests.

Only a few studies have focused on EVs as a novel source of markers for severe malaria. In our group, we used high-throughput proteomics to characterise the protein cargo of MVs released during ECM in P. berghei infected mice ${ }^{[96]}$. The vast majority of identified proteins were host-derived and only a couple were from $P$. berghei. The protein content of MVs released during severe disease was significantly altered compared to that released upon early infection or in uninfected mice. Network analysis showed that proteins with altered abundance during ECM were associated with CM pathogenesis. Two of these proteins, carbonic anhydrase I and S100A8 were verified to be associated with CM MV in both murine and clinical samples, highlighting the importance of MV protein content to understand the role of EVs both in severe malaria and as a source of protein markers ${ }^{[96]}$. The protein cargo of MVs obtained from $P$. falciparuminfected individuals was later investigated by Antwi-Baffour and colleagues ${ }^{[97]}$, although cases with severe malaria were not investigated. The study identified several different host-derived proteins in infected and non-infected human subjects, as well as parasite-derived proteins in infected samples. Nonetheless, the results remained primarily descriptive and no diagnostic marker was actually proposed. More recently, proteomics was applied to identify novel potential biomarkers of $P$. vivax liver stage infection ${ }^{[98]}$. By taking advantage of a human liver-chimeric mouse model, plasma EVs obtained after $P$. vivax infection were studied to identify potential liver-stage expressed parasite proteins that could be indicative of infection. Among mouse and human proteins, they also identified parasite proteins showing variable distribution in abundance over different time points post-infection, indicating that parasite proteins contained within EVs vary with parasite developmental stages, supporting their potential role as a source of biomarkers ${ }^{[98]}$. In mice and human studies, there has been a consistent indication of EVs' importance in the role of malaria pathogenesis and their potential as markers for disease severity; however, more research is required to confirm the potential of these EVs derived protein as biomarkers for severe malaria. 
Within the groups of non-coding RNAs, miRNA are now considered promising biomarkers in many pathological conditions ${ }^{[99,100]}$ due to their stability in various bodily fluids such as saliva, serum, plasma and CSF, and their role in gene expression regulation ${ }^{[101-106]}$. One of the advantages of studying EV-associated miRNAs is their particular stability as they are protected within a plasma membrane ${ }^{[107]}$. These short, noncoding RNA molecules display critical regulatory functions as they are involved in nearly all physiological processes such as cellular differentiation, proliferation, metabolism, development, and homeostasis ${ }^{[108,109]}$.

As previously mentioned, a large portion of EV miRNA studies are focused on cancers, which have shown the significance of identifying cargo miRNA. In a 2019 clinical cancer study, miRNA from whole plasma, EVs and EV-free plasma from lung adenocarcinoma and granuloma patients were evaluated. The study determined that whole plasma, EVs and EV-free plasma had differing miRNA expression profiles and the prediction performance of EVs was better than EV-free plasma. Plasma was the best predictor however, due to the lack of knowledge in storage and processing techniques of $\mathrm{EVs}^{[110]}$. Elevated levels of plasma EVs have since been observed in patients affected by various forms of cancer compared to healthy subjects and, interestingly, these levels decreased upon removal of the tumour, simultaneously decreasing tumour specific miRNA profiles within the plasma $\mathrm{EVs}^{[111,112]}$, which provides a further link between cancer and increased EV production. Similar results were demonstrated in patients with autoimmune, infectious and cardiovascular diseases, and neurological disorders ${ }^{[113-116]}$.

Next-generation sequencing technology is the recommended, standard approach when investigating the miRNA content of EVs for novel biomarker identification ${ }^{[117]}$. Accumulated sequencing data suggest the potential for miRNAs as diagnostic and prognostic markers, as well as for parasitic diseases caused by platyhelminths, arthropods, and protozoa, including Plasmodium spp. ${ }^{[118]}$. When analysing EVs derived from helminth parasites (Trichuris muris), the content was sequenced using the HiSeq 500 system, identifying 56 miRNA, 22 of which were novel ${ }^{[119]}$. A similar study looking at hookworms using the NextSeq 500 system identified 52 miRNA, many of which were found to be involved in inflammation regulation when mapped to mouse genes ${ }^{[120]}$.

One of the first studies on miRNA in Plasmodium infection suggested that this parasite did not have specific miRNAs but rather, takes advantage of the transcriptional machinery within RBCs for the activation and suppression of gene expression ${ }^{[121]}$. This study also identified miR-451 as highly expressed in iRBCs, although its accumulation was not associated with malaria infection ${ }^{[121]}$.

A study from Thailand observed, for the first time, lower expression of miR-451 and miR-16 in the plasma of adults infected with malaria, suggesting their role as biomarkers for malaria infection, especially in Plasmodium vivax infected individuals ${ }^{[122]}$. However, a large portion of transcriptomic studies have been performed using murine models focusing on the host's response to infection ${ }^{[123-125]}$, or using in vitro systems to target a specific cell-type. For instance, let-7i, miR-27a, and miR-150 were found to be over-expressed in the brain of CM-mice but not in non-CM animals ${ }^{[124]}$. Overexpression of these miRNA during infection may be essential for the instigation of neurological syndromes by regulating their downstream targets, thus having a potential regulatory role in the pathogenesis of severe malaria, as well as being targets for therapeutic intervention ${ }^{[124]}$.

Similar to iRBCs, EVs from iRBCs display higher levels of miR-451a and let-7b when compared to nRBCs, and once miR-451a within EVs is engulfed by endothelial cells, their gene expression and barrier properties are affected, which may then lead to vascular dysfunction, making the miRNA a possible target for therapeutic intervention ${ }^{[52]}$. Using a more complex model, our group analysed plasma EVs from mice with $\mathrm{CM}$ and found that the miRNAs from malaria EVs played a regulatory role in severe malaria pathogenesis. miR-146a levels were higher and miR-193b levels lower in plasma-derived EVs while miR-205, miR-215, 
and miR-467a were all elevated in brain tissue from CM mice when compared to non-infected or nonCM infected mice ${ }^{[126]}$. This difference in miRNA profiles suggests that miRNA present in circulation could have different functions from those present in tissues. Further investigation to verify the potential of these EVs derived miRNA as biomarkers for the cerebral syndrome using both experimental models and clinical samples will be necessary.

\section{CONCLUSION}

In vivo and ex vivo studies point towards a role for EVs in the modulation of disease and the host response. No study has looked at the behaviour of EVs in situ however. Rather than passively transferred EVs, animal models utilising both transgenic parasites and transgenic host cells expressing tags that can be traced, combined with high-resolution imaging in the animal, will allow us to truly understand the complex involvement of EVs with their target cells. For instance, recent work used high-resolution microscopy to visualise circulating EVs in zebrafish embryos using a tissue-specific expression of genetically encoded markers of EVs. This approach will allow us to not only decipher the role of EVs in physiology including cargo delivery but also, to assess the effects of disease or treatment on EVs release and function ${ }^{[127]}$.

In addition, although evidence confirming the importance of EVs as a source of biomarkers are scattered, they also highlight a number of questions and unsolved problems. Indeed, most of the work performed so far still lack validation steps and clinical studies remain scarce. These limitations are such that most studies remain mainly descriptive and hamper the process of biomarker validation and implementation into clinical practice. In-depth investigations should also be carried out to understand the mechanisms of protein and miRNA packaging into EVs, as well as the signals involved in cell targeting. Deciphering these processes will contribute to the selection of highly specific biomarkers for larger validation studies. While biomarker studies applied to severe malaria and EVs are still in their infancy, there is hope for this field to provide novel strategies to fight severe malaria in the future.

\section{DECLARATIONS}

\section{Authors' contributions}

Conceived and designed the review: Cheng IS, Sealy BC, Tiberti N, Combes V

Made equal contribution to the writing of the sections: Cheng IS, Sealy BC

Provided feedback for manuscript revision: Cheng IS, Sealy BC

Read and approved the final manuscript: Cheng IS, Sealy BC, Tiberti N, Combes V

\section{Availability of data and materials}

Not applicable.

\section{Financial support and sponsorship}

Combes V was supported by the University of Technology, Faculty of Science grant. Cheng IS and Sealy BC were supported by the Australian Government Research Training Program Stipend. Tiberti N was supported by the Italian Ministry of Health "Fondi Ricerca Corrente - Linea 1 Progetto 3" to IRCCS. Sacro Cuore Don Calabria Hospital.

\section{Conflicts of interest}

All authors declared that there are no conflicts of interest.

\section{Ethical approval and consent to participate}

Not applicable. 


\section{Consent for publication}

Not applicable.

\section{Copyright}

(c) The Author(s) 2020.

\section{REFERENCES}

1. Organization WH. World malaria report 2019. 2019. Available from https:/www.who.int/publications-detail/world-malaria-report-2019 [Last accessed on 6 May 2020]

2. Murray CJL, Rosenfeld LC, Lim SS, Andrews KG, Foreman KJ, et al. Global malaria mortality between 1980 and 2010 : a systematic analysis. Lancet 2012;379:413-31.

3. Bangirana P, Opoka RO, Boivin MJ, Idro R, Hodges JS, et al. Severe malarial anemia is associated with long-term neurocognitive impairment. Clin Infect Dis 2014;59:336-44.

4. Storm J, Craig AG. Pathogenesis of cerebral malaria--inflammation and cytoadherence. Front Cell Infect Microbiol 2014;4:100.

5. Organization WH. Management of severe malaria: a practical handbook. apps.who.int; 2012. Available from https://apps.who.int/iris/ handle/10665/79317 [Last accessed on 6 May 2020]

6. Jakeman GN, Saul A, Hogarth WL, Collins WE. Anaemia of acute malaria infections in non-immune patients primarily results from destruction of uninfected erythrocytes. Parasitology 1999;119:127-33.

7. Lamikanra AA, Brown D, Potocnik A, Casals-Pascual C, Langhorne J, et al. Malarial anemia: of mice and men. Blood 2007;110:18-28.

8. Vainieri ML, Blagborough AM, MacLean AL, Haltalli MLR, Ruivo N, et al. Systematic tracking of altered haematopoiesis during sporozoite-mediated malaria development reveals multiple response points. Open Biol 2016;6:160038.

9. Razakandrainibe R, Combes V, Grau GE, Jambou R. Crossing the wall: the opening of endothelial cell junctions during infectious diseases. Int J Biochem Cell Biol 2013;45:1165-73.

10. Dorovini-Zis K, Schmidt K, Huynh H, Fu W, Whitten RO, et al. The neuropathology of fatal cerebral malaria in malawian children. Am J Pathol 2011;178:2146-58.

11. Taylor TE, Fu WJ, Carr RA, Whitten RO, Mueller JS, et al. Differentiating the pathologies of cerebral malaria by postmortem parasite counts. Nat Med 2004;10:143-5.

12. Ponsford MJ, Medana IM, Prapansilp P, Hien TT, Lee SJ, et al. Sequestration and microvascular congestion are associated with coma in human cerebral malaria. J Infect Dis 2012;205:663-71.

13. Combes V, Guillemin GJ, Chan-Ling T, Hunt NH, Grau GER. The crossroads of neuroinflammation in infectious diseases: endothelial cells and astrocytes. Trends Parasitol 2012;28:311-9.

14. Dunst J, Kamena F, Matuschewski K. Cytokines and chemokines in cerebral malaria pathogenesis. Front Cell Infect Microbiol 2017;7:324.

15. Wassmer SC, Grau GER. Platelets as pathogenetic effectors and killer cells in cerebral malaria. Expert Rev Hematol 2016;9:515-7.

16. Chimalizeni Y, Kawaza K, Taylor T, Molyneux M. The platelet count in cerebral malaria, is it useful to the clinician? Am J Trop Med Hyg 2010;83:48-50.

17. Gérardin P, Rogier C, Ka AS, Jouvencel P, Brousse V, et al. Prognostic value of thrombocytopenia in African children with falciparum malaria. Am J Trop Med Hyg 2002;66:686-91.

18. Grau GE, Mackenzie CD, Carr RA, Redard M, Pizzolato G, et al. Platelet accumulation in brain microvessels in fatal pediatric cerebral malaria. J Infect Dis 2003;187:461-6.

19. Wassmer SC, Taylor T, Maclennan CA, Kanjala M, Mukaka M, et al. Platelet-induced clumping of Plasmodium falciparum-infected erythrocytes from Malawian patients with cerebral malaria-possible modulation in vivo by thrombocytopenia. J Infect Dis 2008;197:72-8.

20. Barbier M, Faille D, Loriod B, Textoris J, Camus C, et al. Platelets alter gene expression profile in human brain endothelial cells in an in vitro model of cerebral malaria. PLoS One 2011;6:e19651.

21. Wassmer SC, Combes V, Candal FJ, Juhan-Vague I, Grau GE. Platelets potentiate brain endothelial alterations induced by Plasmodium falciparum. Infect Immun 2006;74:645-53.

22. McMorran BJ, Wieczorski L, Drysdale KE, Chan JA, Huang HM, et al. Platelet factor 4 and Duffy antigen required for platelet killing of Plasmodium falciparum. Science 2012;338:1348-51.

23. Peyron F, Polack B, Lamotte D, Kolodie L, Ambroise-Thomas P. Plasmodium falciparum growth inhibition by human platelets in vitro. Parasitology 1989;99:317-22.

24. Aggrey AA, Srivastava K, Ture S, Field DJ, Morrell CN. Platelet induction of the acute-phase response is protective in murine experimental cerebral malaria. J Immunol 2013;190:4685-91.

25. Medana IM, Turner GDH. Human cerebral malaria and the blood-brain barrier. Int J Parasitol 2006;36:555-68.

26. Medana IM, Day NP, Hien TT, Mai NTH, Bethell D, et al. Axonal injury in cerebral malaria. Am J Pathol 2002;160:655-66.

27. Adams S, Brown H, Turner G. Breaking down the blood-brain barrier: signaling a path to cerebral malaria? Trends Parasitol 2002;18:360-6.

28. Beare NAV, Harding SP, Taylor TE, Lewallen S, Molyneux ME. Perfusion abnormalities in children with cerebral malaria and malarial retinopathy. J Infect Dis 2009;199:263-71.

29. Kampondeni SD, Birbeck GL, Seydel KB, Beare NA, Glover SJ, et al. Noninvasive measures of brain edema predict outcome in pediatric 
cerebral malaria. Surg Neurol Int 2018;9:53.

30. Seydel KB, Kampondeni SD, Valim C, Potchen MJ, Milner DA, et al. Brain swelling and death in children with cerebral malaria. N Engl J Med 2015;372:1126-37.

31. Schindler SM, Little JP, Klegeris A. Microparticles: a new perspective in central nervous system disorders. Biomed Res Int 2014;2014:756327.

32. Schofield L, Grau GE. Immunological processes in malaria pathogenesis. Nat Rev Immunol 2005;5:722-35.

33. Doyle LM, Wang MZ. Overview of extracellular vesicles, their origin, composition, purpose, and methods for exosome isolation and analysis. Cells 2019;8:727.

34. Mantel PY, Marti M. The role of extracellular vesicles in Plasmodium and other protozoan parasites. Cell Microbiol 2014;16:344-54.

35. Torrecilhas AC, Schumacher RI, Alves MJM, Colli W. Vesicles as carriers of virulence factors in parasitic protozoan diseases. Microbes Infect 2012;14:1465-74.

36. Combes V, Taylor TE, Juhan-Vague I, Mège JL, Mwenechanya J, et al. Circulating endothelial microparticles in malawian children with severe falciparum malaria complicated with coma. JAMA 2004;291:2542-4.

37. Campos FMF, Franklin BS, Teixeira-Carvalho A, Filho ALS, de Paula SCO, et al. Augmented plasma microparticles during acute Plasmodium vivax infection. Malar J 2010;9:327.

38. Nantakomol D, Dondorp AM, Krudsood S, Udomsangpetch R, Pattanapanyasat K, et al. Circulating red cell-derived microparticles in human malaria. J Infect Dis 2011;203:700-6.

39. Pankoui Mfonkeu JB, Gouado I, Fotso Kuaté H, Zambou O, Amvam Zollo PH, et al. Elevated cell-specific microparticles are a biological marker for cerebral dysfunctions in human severe malaria. PLoS One 2010;5:e13415.

40. Martin-Jaular L, Nakayasu ES, Ferrer M, Almeida IC, Del Portillo HA. Exosomes from Plasmodium yoelii-infected reticulocytes protect mice from lethal infections. PLoS One 2011;6:e26588.

41. Bridges DJ, Bunn J, van Mourik JA, Grau G, Preston RJS, et al. Rapid activation of endothelial cells enables Plasmodium falciparum adhesion to platelet-decorated von Willebrand factor strings. Blood 2010;115:1472-4.

42. El-Assaad F, Wheway J, Mitchell AJ, Lou J, Hunt NH, et al. Cytoadherence of Plasmodium berghei-infected red blood cells to murine brain and lung microvascular endothelial cells in vitro. Infect Immun 2013;81:3984-91.

43. Jambou R, El-Assaad F, Combes V, Grau GE. In vitro culture of Plasmodium berghei-ANKA maintains infectivity of mouse erythrocytes inducing cerebral malaria. Malar J 2011;10:346.

44. Khaw LT, Ball HJ, Golenser J, Combes V, Grau GE, et al. Endothelial cells potentiate interferon- $\gamma$ production in a novel tripartite culture model of human cerebral malaria. PLoS One 2013;8:e69521.

45. Wassmer SC, Lépolard C, Traoré B, Pouvelle B, Gysin J, et al. Platelets reorient Plasmodium falciparum-infected erythrocyte cytoadhesion to activated endothelial cells. J Infect Dis 2004;189:180-9.

46. Canfield SG, Stebbins MJ, Morales BS, Asai SW, Vatine GD, et al. An isogenic blood-brain barrier model comprising brain endothelial cells, astrocytes, and neurons derived from human induced pluripotent stem cells. J Neurochem 2017;140:874-88.

47. Cho H, Seo JH, Wong KHK, Terasaki Y, Park J, et al. Three-dimensional blood-brain barrier model for in vitro studies of neurovascular pathology. Sci Rep 2015;5:15222.

48. Helms HC, Abbott NJ, Burek M, Cecchelli R, Couraud PO, et al. In vitro models of the blood-brain barrier: an overview of commonly used brain endothelial cell culture models and guidelines for their use. J Cereb Blood Flow Metab 2016;36:862-90.

49. Faille D, Combes V, Mitchell AJ, Fontaine A, Juhan-Vague I, et al. Platelet microparticles: a new player in malaria parasite cytoadherence to human brain endothelium. FASEB J 2009;23:3449-58.

50. Wassmer SC, Combes V, Grau GER. Platelets and microparticles in cerebral malaria: the unusual suspects. Drug Discov Today Dis Mechanisms 2011;8:e15-23.

51. Sampaio NG, Emery SJ, Garnham AL, Tan QY, Sisquella X, et al. Extracellular vesicles from early stage Plasmodium falciparuminfected red blood cells contain PfEMP1 and induce transcriptional changes in human monocytes. Cell Microbiol 2018;20:e12822.

52. Mantel PY, Hjelmqvist D, Walch M, Kharoubi-Hess S, Nilsson S, et al. Infected erythrocyte-derived extracellular vesicles alter vascular function via regulatory Ago2-miRNA complexes in malaria. Nat Commun 2016;7:12727.

53. Wang Z, Xi J, Hao X, Deng W, Liu J, et al. Red blood cells release microparticles containing human argonaute 2 and miRNAs to target genes of Plasmodium falciparum. Emerg Microbes Infect 2017;6:e75.

54. Couper KN, Barnes T, Hafalla JCR, Combes V, Ryffel B, et al. Parasite-derived plasma microparticles contribute significantly to malaria infection-induced inflammation through potent macrophage stimulation. PLoS Pathog 2010;6:e1000744.

55. Mantel PY, Hoang AN, Goldowitz I, Potashnikova D, Hamza B, et al. Malaria-infected erythrocyte-derived microvesicles mediate cellular communication within the parasite population and with the host immune system. Cell Host Microbe 2013;13:521-34.

56. Sisquella X, Ofir-Birin Y, Pimentel MA, Cheng L, Abou Karam P, et al. Malaria parasite DNA-harbouring vesicles activate cytosolic immune sensors. Nat Commun 2017;8:1985.

57. Yu X, Cai B, Wang M, Tan P, Ding X, et al. Cross-regulation of two type i interferon signaling pathways in plasmacytoid dendritic cells controls anti-malaria immunity and host mortality. Immunity 2016;45:1093-107.

58. Demarta-Gatsi C, Rivkin A, Di Bartolo V, Peronet R, Ding S, et al. Histamine releasing factor and elongation factor 1 alpha secreted via malaria parasites extracellular vesicles promote immune evasion by inhibiting specific T cell responses. Cell Microbiol 2019;21:e13021.

59. Regev-Rudzki N, Wilson DW, Carvalho TG, Sisquella X, Coleman BM, et al. Cell-cell communication between malaria-infected red blood cells via exosome-like vesicles. Cell 2013;153:1120-33.

60. Wheway J, Latham SL, Combes V, Grau GER. Endothelial microparticles interact with and support the proliferation of T cells. J Immunol 
2014;193:3378-87.

61. Gasser O, Schifferli JA. Activated polymorphonuclear neutrophils disseminate anti-inflammatory microparticles by ectocytosis. Blood 2004; 104:2543-8.

62. Rhys HI, Dell'Accio F, Pitzalis C, Moore A, Norling LV, et al. Neutrophil microvesicles from healthy control and rheumatoid arthritis patients prevent the inflammatory activation of macrophages. EBioMedicine 2018;29:60-9.

63. Wen B, Combes V, Bonhoure A, Weksler BB, Couraud PO, et al. Endotoxin-induced monocytic microparticles have contrasting effects on endothelial inflammatory responses. PLoS One 2014;9:e91597.

64. Riggle BA, Manglani M, Maric D, Johnson KR, Lee MH, et al. CD8+ T cells target cerebrovasculature in children with cerebral malaria. J Clin Invest 2020;130:1128-38.

65. Poh CM, Howland SW, Grotenbreg GM, Rénia L. Damage to the blood-brain barrier during experimental cerebral malaria results from synergistic effects of CD8+ T cells with different specificities. Infect Immun 2014;82:4854-64.

66. Swanson PA, Hart GT, Russo MV, Nayak D, Yazew T, et al. CD8+ T cells induce fatal brainstem pathology during cerebral malaria via luminal antigen-specific engagement of brain vasculature. PLoS Pathog 2016;12:e1006022.

67. Combes V, Souza JBD, Rénia L, Hunt NH, Grau GE. Cerebral malaria: which parasite? Which model? Drug Discov Today Dis Models 2005;2:141-7.

68. Craig AG, Grau GE, Janse C, Kazura JW, Milner D, et al. The role of animal models for research on severe malaria. PLoS Pathog 2012;8:e1002401.

69. de Souza JB, Hafalla JCR, Riley EM, Couper KN. Cerebral malaria: why experimental murine models are required to understand the pathogenesis of disease. Parasitology 2010;137:755-72.

70. El-Assaad F, Combes V, Grau GE. Experimental models of microvascular immunopathology: the example of cerebral malaria. J Neuroinfect Dis 2014;5.

71. Riley EM, Couper KN, Helmby H, Hafalla JCR, de Souza JB, et al. Neuropathogenesis of human and murine malaria. Trends Parasitol 2010;26:277-8.

72. Amante FH, Stanley AC, Randall LM, Zhou Y, Haque A, et al. A role for natural regulatory T cells in the pathogenesis of experimental cerebral malaria. Am J Pathol 2007;171:548-59.

73. Claser C, Malleret B, Gun SY, Wong AYW, Chang ZW, et al. CD8+ T cells and IFN- $\gamma$ mediate the time-dependent accumulation of infected red blood cells in deep organs during experimental cerebral malaria. PLoS One 2011;6:e18720.

74. Franke-Fayard B, Janse CJ, Cunha-Rodrigues M, Ramesar J, Büscher P, et al. Murine malaria parasite sequestration: CD36 is the major receptor, but cerebral pathology is unlinked to sequestration. Proc Natl Acad Sci U S A 2005;102:11468-73.

75. Strangward P, Haley MJ, Shaw TN, Schwartz JM, Greig R, et al. A quantitative brain map of experimental cerebral malaria pathology. PLoS Pathog 2017;13:e1006267.

76. Combes V, Coltel N, Alibert M, van Eck M, Raymond C, et al. ABCA1 gene deletion protects against cerebral malaria: potential pathogenic role of microparticles in neuropathology. Am J Pathol 2005;166:295-302.

77. El-Assaad F, Wheway J, Hunt NH, Grau GER, Combes V. Production, fate and pathogenicity of plasma microparticles in murine cerebral malaria. PLoS Pathog 2014;10:e1003839.

78. Penet MF, Abou-Hamdan M, Coltel N, Cornille E, Grau GE, et al. Protection against cerebral malaria by the low-molecular-weight thiol pantethine. Proc Natl Acad Sci U S A 2008;105:1321-6.

79. Kuhn SM, McCarthy AE. Paediatric malaria: what do paediatricians need to know? Paediatr Child Health 2006;11:349-54.

80. White NJ, Pukrittayakamee S, Hien TT, Faiz MA, Mokuolu OA, et al. Malaria. Lancet 2014;383:723-35.

81. Shrivastava SK, Dalko E, Delcroix-Genete D, Herbert F, Cazenave PA, et al. Uptake of parasite-derived vesicles by astrocytes and microglial phagocytosis of infected erythrocytes may drive neuroinflammation in cerebral malaria. Glia 2017;65:75-92.

82. Martín-Jaular L, de Menezes-Neto A, Monguió-Tortajada M, Elizalde-Torrent A, Díaz-Varela M, et al. Spleen-dependent immune protection elicited by $\mathrm{CpG}$ adjuvanted reticulocyte-derived exosomes from malaria infection is associated with changes in T cell subsets' distribution. Front Cell Dev Biol 2016;4:131.

83. Nantakomol D, Chimma P, Day NP, Dondorp AM, Combes V, et al. Quantitation of cell-derived microparticles in plasma using flow rate based calibration. Southeast Asian J Trop Med Public Health 2008;39:146-53.

84. Babatunde KA, Yesodha Subramanian B, Ahouidi AD, Martinez Murillo P, Walch M, et al. Role of extracellular vesicles in cellular cross talk in malaria. Front Immunol 2020;11:22.

85. Sahu PK, Satpathi S, Behera PK, Mishra SK, Mohanty S, et al. Pathogenesis of cerebral malaria: new diagnostic tools, biomarkers, and therapeutic approaches. Front Cell Infect Microbiol 2015;5:75.

86. Varo R, Crowley VM, Sitoe A, Madrid L, Serghides L, et al. Adjunctive therapy for severe malaria: a review and critical appraisal. Malar J 2018;17:47.

87. Adukpo S, Kusi KA, Ofori MF, Tetteh JKA, Amoako-Sakyi D, et al. High plasma levels of soluble intercellular adhesion molecule (ICAM)-1 are associated with cerebral malaria. PLoS One 2013;8:e84181.

88. Casals-Pascual C, Idro R, Gicheru N, Gwer S, Kitsao B, et al. High levels of erythropoietin are associated with protection against neurological sequelae in African children with cerebral malaria. Proc Natl Acad Sci U S A 2008;105:2634-9.

89. Conroy AL, Lafferty EI, Lovegrove FE, Krudsood S, Tangpukdee N, et al. Whole blood angiopoietin-1 and -2 levels discriminate cerebral and severe (non-cerebral) malaria from uncomplicated malaria. Malar J 2009;8:295.

90. Thakur K, Vareta J, Carson K, Taylor T, Sullivan D. Performance of cerebrospinal fluid (CSF) plasmodium falciparum histidine-rich protein-2 (pfHRP-2) in prediction of death in cerebral malaria (I10-2.005) 2014. Available from https://n.neurology.org/content $/ 82 / 10$ 
Supplement/110-2.005 [Last accessed on 6 May 2020]

91. Antwi-Baffour S, Malibha-Pinchbeck M, Stratton D, Jorfi S, Lange S, et al. Plasma mEV levels in Ghanain malaria patients with low parasitaemia are higher than those of healthy controls, raising the potential for parasite markers in mEVs as diagnostic targets. J Extracell Vesicles 2020;9:1697124.

92. Hede MS, Fjelstrup S, Lötsch F, Zoleko RM, Klicpera A, et al. Detection of the malaria causing plasmodium parasite in saliva from infected patients using topoisomerase I activity as a biomarker. Sci Rep 2018;8:4122.

93. Krampa FD, Aniweh Y, Awandare GA, Kanyong P. Recent progress in the development of diagnostic tests for malaria. Diagnostics (Basel) 2017;7:54.

94. Choi DS, Kim DK, Kim YK, Gho YS. Proteomics, transcriptomics and lipidomics of exosomes and ectosomes. Proteomics 2013;13:1554-71.

95. Abdi A, Yu L, Goulding D, Rono MK, Bejon P, et al. Proteomic analysis of extracellular vesicles from a Plasmodium falciparum Kenyan clinical isolate defines a core parasite secretome. [version 2; peer review: 2 approved, 1 approved with reservations]. Wellcome Open Res 2017;2:50.

96. Tiberti N, Latham SL, Bush S, Cohen A, Opoka RO, et al. Exploring experimental cerebral malaria pathogenesis through the characterisation of host-derived plasma microparticle protein content. Sci Rep 2016;6:37871.

97. Antwi-Baffour S, Adjei JK, Agyemang-Yeboah F, Annani-Akollor M, Kyeremeh R, et al. Proteomic analysis of microparticles isolated from malaria positive blood samples. Proteome Sci 2016;15:5.

98. Gualdrón-López M, Flannery EL, Kangwanrangsan N, Chuenchob V, Fernandez-Orth D, et al. Characterization of plasmodium vivax proteins in plasma-derived exosomes from malaria-infected liver-chimeric humanized Mice. Front Microbiol 2018;9:1271.

99. Hanna J, Hossain GS, Kocerha J. The Potential for microRNA therapeutics and clinical research. Front Genet 2019;10:478.

100. Saliminejad K, Khorram Khorshid HR, Soleymani Fard S, Ghaffari SH. An overview of microRNAs: biology, functions, therapeutics, and analysis methods. J Cell Physiol 2019;234:5451-65.

101. Chen SY, Wang Y, Telen MJ, Chi JT. The genomic analysis of erythrocyte microRNA expression in sickle cell diseases. PLoS One 2008;3:e2360.

102. Chen X, Ba Y, Ma L, Cai X, Yin Y, et al. Characterization of microRNAs in serum: a novel class of biomarkers for diagnosis of cancer and other diseases. Cell Res 2008;18:997-1006.

103. Hammond SM. An overview of microRNAs. Adv Drug Deliv Rev 2015;87:3-14.

104. Mitchell PS, Parkin RK, Kroh EM, Fritz BR, Wyman SK, et al. Circulating microRNAs as stable blood-based markers for cancer detection. Proc Natl Acad Sci U S A 2008;105:10513-8.

105. Reid G, Kirschner MB, van Zandwijk N. Circulating microRNAs: association with disease and potential use as biomarkers. Crit Rev Oncol Hematol 2011;80:193-208.

106. Wang W, Li R, Meng M, Wei C, Xie Y, et al. MicroRNA profiling of CD3+ CD56+ cytokine-induced killer cells. Sci Rep 2015;5:9571.

107. Babatunde KA, Mbagwu S, Hernández-Castañeda MA, Adapa SR, Walch M, et al. Malaria infected red blood cells release small regulatory RNAs through extracellular vesicles. Sci Rep 2018;8:884.

108. Bartel DP. MicroRNAs: genomics, biogenesis, mechanism, and function. Cell 2004;116:281-97.

109. Wang J, Sen S. MicroRNA functional network in pancreatic cancer: from biology to biomarkers of disease. J Biosci 2011;36:481-91.

110. Chen X, Jin Y, Feng Y. Evaluation of plasma extracellular vesicle microRNA signatures for lung adenocarcinoma and granuloma with monte-carlo feature selection method. Front Genet 2019;10:367.

111. Sartori MT, Della Puppa A, Ballin A, Saggiorato G, Bernardi D, et al. Prothrombotic state in glioblastoma multiforme: an evaluation of the procoagulant activity of circulating microparticles. J Neurooncol 2011;104:225-31.

112. Zwicker JI, Liebman HA, Neuberg D, Lacroix R, Bauer KA, et al. Tumor-derived tissue factor-bearing microparticles are associated with venous thromboembolic events in malignancy. Clin Cancer Res 2009;15:6830-40.

113. da Silva EFR, Fonseca FAH, França CN, Ferreira PRA, Izar MCO, et al. Imbalance between endothelial progenitors cells and microparticles in HIV-infected patients naive for antiretroviral therapy. AIDS 2011;25:1595-601.

114. Pelletier F, Garnache-Ottou F, Angelot F, Biichlé S, Vidal C, et al. Increased levels of circulating endothelial-derived microparticles and small-size platelet-derived microparticles in psoriasis. J Invest Dermatol 2011;131:1573-6.

115. Stępień E, Stankiewicz E, Zalewski J, Godlewski J, Zmudka K, et al. Number of microparticles generated during acute myocardial infarction and stable angina correlates with platelet activation. Arch Med Res 2012;43:31-5.

116. Xue S, Cai X, Li W, Zhang Z, Dong W, et al. Elevated plasma endothelial microparticles in Alzheimer's disease. Dement Geriatr Cogn Disord 2012;34:174-80.

117. Bondar G, Xu W, Elashoff D, Li X, Faure-Kumar E, et al. Comparing NGS and NanoString platforms in peripheral blood mononuclear cell transcriptome profiling for advanced heart failure biomarker development. J Biol Methods 2020;7:e123.

118. Manzano-Román R, Siles-Lucas M. MicroRNAs in parasitic diseases: potential for diagnosis and targeting. Mol Biochem Parasitol 2012;186:81-6.

119. Eichenberger RM, Talukder MH, Field MA, Wangchuk P, Giacomin P, et al. Characterization of Trichuris muris secreted proteins and extracellular vesicles provides new insights into host-parasite communication. J Extracell Vesicles 2018;7:1428004.

120. Eichenberger RM, Ryan S, Jones L, Buitrago G, Polster R, et al. Hookworm secreted extracellular vesicles interact with host cells and prevent inducible colitis in mice. Front Immunol 2018;9:850.

121. Xue X, Zhang Q, Huang Y, Feng L, Pan W. No miRNA were found in Plasmodium and the ones identified in erythrocytes could not be correlated with infection. Malar J 2008;7:47.

122. Chamnanchanunt S, Kuroki C, Desakorn V, Enomoto M, Thanachartwet V, et al. Downregulation of plasma miR-451 and miR-16 in 
Plasmodium vivax infection. Exp Parasitol 2015;155:19-25.

123. Capuccini B, Lin J, Talavera-López C, Khan SM, Sodenkamp J, et al. Transcriptomic profiling of microglia reveals signatures of cell activation and immune response, during experimental cerebral malaria. Sci Rep 2016;6:39258.

124. El-Assaad F, Hempel C, Combes V, Mitchell AJ, Ball HJ, et al. Differential microRNA expression in experimental cerebral and noncerebral malaria. Infect Immun 2011;79:2379-84.

125. Lin JW, Sodenkamp J, Cunningham D, Deroost K, Tshitenge TC, et al. Signatures of malaria-associated pathology revealed by highresolution whole-blood transcriptomics in a rodent model of malaria. Sci Rep 2017;7:41722.

126. Cohen A, Zinger A, Tiberti N, Grau GER, Combes V. Differential plasma microvesicle and brain profiles of microRNA in experimental cerebral malaria. Malar J 2018;17:192.

127. Verweij FJ, Hyenne V, Van Niel G, Goetz JG. Extracellular vesicles: catching the light in zebrafish. Trends Cell Biol 2019;29:770-6. 